\title{
The bipolar spectrum
}

\author{
JULES ANGST
}

\begin{abstract}
Summary The two-dimensional bipolar spectrum described here comprises a continuum of severity from normal to psychotic and a continuum from depression, via three bipolar subgroups to mania. This combination of dimensional and categorical principles for classifying mood disorders may help alleviate the problems of underdiagnosis and undertreatment of bipolar disorders.
\end{abstract}

\section{Declaration of interest None.}

Depression is very distressful, prompts the depressed person to seek treatment and is relatively easy to diagnose. Hypomania, on the other hand, is often perceived as normal well-being and tends not to be reported. There is wide agreement as to the difficulty of identifying hypomania in bipolar II and minor bipolar disorders: patients with bipolar depression report that the recognition of their disorder was delayed by as much as $8-10$ years. Unipolar depression, which is defined by the absence of hypomania or mania, remains an uncertain diagnosis lifelong. Over decades of recurrent depressive illness, bipolar disorder may manifest at any time: a lifelong follow-up of patients hospitalised showed a persistent risk of diagnostic change to bipolar disorder of $1.25 \%$ per year of observation.

Bipolar affective disorder is a more severe disorder than major depression, as measured by higher lifelong recurrence and greater comorbidity with psychiatric disorders, especially anxiety and secondary substance use disorders. In addition it is associated with serious somatic disorders such as diabetes, hypertension and cardiovascular disease. This explains the correspondingly higher mortality rates among people with bipolar disorder, although the suicide risk is lower in type I bipolar disorder than in depression (Ösby et al, 2001).

Correct diagnosis of bipolar illness is essential for appropriate treatment, especially long-term secondary prophylaxis. As a consequence of their severity, unrecognised bipolar disorders lead, moreover, to higher costs than major depression, but these can be considerably reduced by early diagnosis and treatment, as recently shown by McCombs et al (2006).

The underdiagnosis of mood disorders, especially bipolar disorders, is not confined to the clinical setting but may also apply to traditional epidemiological studies, which found lifetime prevalence rates of $0.5-2 \%$. Some recent studies (Kessler et al, 2003; Lieb, 2006) comprising two or three interview waves have described growing lifetime prevalence rates for both major depressive episodes ( $19 \%$ to $24 \%)$ and for bipolar disorder types I and II together (about $2 \%$ to $4 \%)$. An important question, then, is what proportion of patients with major depression should in fact be diagnosed as having bipolar disorder: is it one-fifth or one-tenth as generally reported, or as many as half, as we have found?

\section{BIPOLAR SPECTRUM: A MODEL FOR RESEARCH AND CLINICAL PRACTICE}

The development of a validated bipolar spectrum concept can provide a more differentiated research and treatment model for affective disorders and may help reduce the underrecognition of bipolarity.

A dimensional concept (from normal to pathological) was proposed by Kretschmer in 1921 for schizophrenia (schizothymic schizoid - schizophrenic) and for affective disorders (cyclothymic temperament cycloid 'psychopathy' - manic-depressive disorder) as well as by Bleuler (1922). The term 'spectrum' was first used in psychiatry in 1968 for the schizophrenia spectrum,

Fig. I Two-dimensional mood/affective spectrum (does not include schizoaffective disorder, as a transition to the schizophrenic spectrum). The precise relationship of personality disorders to the disease spectra is uncertain and an unsolved general problem of psychiatric classification. BP-I (-II), bipolar-I disorder type I (II); D, major depression, d, minor depression; M, mania; m, hypomania; MDD, major depressive disorder; RBD, recurrent brief depression; sx, symptoms

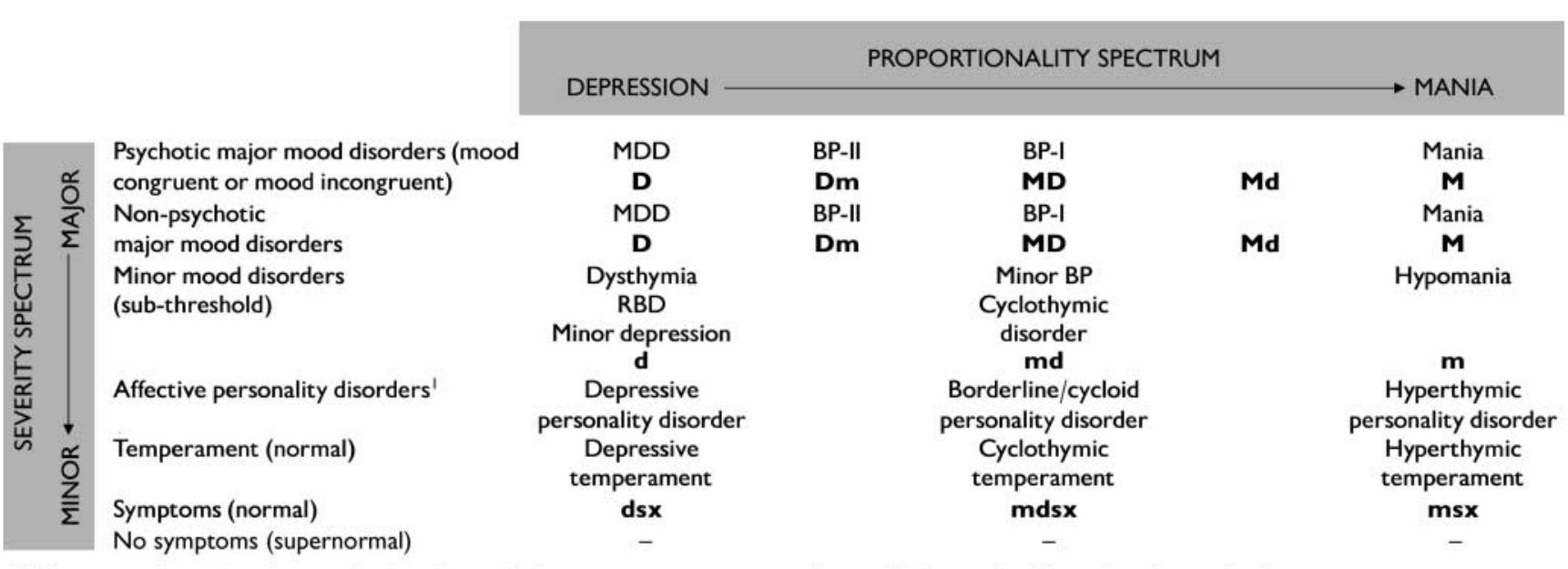

I. The precise relationship of personality disorders to the disease spectra is uncertain and an unsolved general problem of psychiatric classification. 
which integrated schizoid personalities (Kety et al, 1968). In 1977 Akiskal proposed a cyclothymic-bipolar spectrum and in 1981 Klerman suggested a mania spectrum (Akiskal, 1977; Klerman, 1981).

Today the term 'bipolar spectrum' is mainly used in two complementary senses (Fig. 1):

(a) a spectrum of severity, which embraces psychotic and non-psychotic major and minor bipolar disorders (including bipolar dysthymia, recurrent brief and minor depressions), cyclothymic disorders, hypomania and, at its broadest, even borderline disorders and cyclothymic temperament;

(b) a proportional mood spectrum, which considers the two components mania and depression, first on the level of major mood disorders - major depression (D) bipolar II disorder (Dm), bipolar I disorder (MD), mania with mild depression $(\mathrm{Md})$ and pure mania (M) (Angst, 1978) - and then on the level of minor mood disorders: the corresponding categories are mild depression (d), minor bipolar disorder (md) and hypomania (m).

This proportional model is an extension of Kleist's concept of bipolar disorder as a combination of the two monopolar disorders depression and mania (Kleist, 1937, 1953).This model has proved fruitful not only in incorporating bipolar I and bipolar II disorders but also in differentiating mania with or without mild depression $(\mathrm{Md} / \mathrm{M})$ from bipolar I disorder (MD). Mania is not identical to bipolar I disorder in terms of family history, course and suicide risk, and on the sub-threshold level hypomania is not the same as minor bipolar disorder or cyclothymic disorder in terms of family history and temperament.

Both the above bipolar spectrum concepts are dimensional in nature, having no natural categorical subgroups. Epidemiological and clinical studies have demonstrated the continuous distribution of depressive and hypomanic/manic symptoms and syndromes from normal to pathological. Psychiatric symptoms in consulting populations have been shown to be dimensional (Goldberg, 2000). Moreover, in a 20-year follow-up, patients with type I and type II bipolar disorder were found to spend about half the time in sub-threshold affective conditions, and these were dimensional, involving the full range of symptom severity of depression and hypomania (Judd et al,
2003). Most healthy people report depressive and hypomanic symptoms and many are identifiable as manifesting depressive, hypomanic and cyclothymic temperaments, which appear to predispose to the respective affective disorders and personality disorders (see Fig. 1). Only about $15 \%$ of the population report no such symptom over their lifetime and are 'super-normal', with very low scores for vegetative lability and neuroticism.

\section{CASENESS}

The dimensional nature of the mood spectrum raises the question of the correct cut-off levels for caseness (Wing et al, 1978). The current concept of bipolar-II disorder requires a diagnosis of hypomania in addition to major depression. However, the definition of hypomania is the subject of much controversy and research. It is generally agreed that the DSM-IV criteria (American Psychiatric Association, 1994) are too strict (not sensitive) and not based on empirical evidence (not validated). All aspects of the definition are under discussion: the quality of the stem questions (criterion A), which are restricted to elevated and irritable mood (i.e. do not consider symptoms and signs of increased activity), the number and nature of symptoms required and the minimum duration of an episode. A duration of under 4 days ( 2 days or 1 day) and the presence of two or three instead of three or four of the seven symptoms of hypomania are now proposed and have been partially validated. As with depression, brief spells of hypomania (1-3 days) are far more common than manifestations lasting 4 days or 1 week. In order to improve the recognition of bipolarity, we have proposed a sub-diagnostic concept consisting of a few hypomanic symptoms of brief duration associated with a lifetime diagnosis of depression.

What we need today is an empirically validated, sensitive definition of hypomania, which will allow early recognition of major and minor bipolar disorders. Promising modern screening instruments for the self-assessment of hypomanic symptoms have now been developed such as the Mood Disorder Questionnaire (Hirschfeld et al, 2000) and the Hypomania Checklist-32 (Angst et al, 2005), but there is still no gold standard for valid cut-off points for caseness on these continuous measures: that would depend on a validated definition of hypomania, which is still lacking. A similar problem is present in measures of temperament, where clear distinctions between depressive, hyperthymic and cycloid ('cyclothymic') personality disorders are desirable.

\section{THE FUTURE}

It may take longer than hoped to develop better-validated diagnostic criteria: apart from genetic data, long-term follow-up studies over at least 10 years are needed in order to approach diagnostic classifications that can be used as gold standards. For this reason the forthcoming ICD-11 and DSM-V may again have to give us definitions with a limited half-life.

Too many studies, especially in epidemiology, have used methods tailored and restricted to the current DSM-IV diagnostic concepts and have not collected additional data which would have allowed those concepts to be questioned - and too many journals and reviewers hesitate to accept papers that deviate methodologically from the current diagnostic conservatism. At the other extreme, the promising bipolar spectrum concept can be discredited by uncritical generalisations and over-inclusiveness, for instance by taking for granted that cyclothymic personality or borderline disorders are validated elements of the bipolar spectrum. These hypotheses may be correct, but we need much more genetic and follow-up evidence to support them.

We can safely assume that the prevalence of bipolar disorders is seriously under-reported and that the burden of bipolar disorder, estimated by the World Health Organization to be much lower than that of depression, will as a consequence have to be reassessed.

The mood spectrum is also embedded in the spectrum of functional psychoses, including schizophrenia and schizoaffective and affective disorders. There is growing clinical evidence that the spectrum approach, with its dimensional nature, offers a real alternative to the traditional Kraepelinian dichotomy of schizophrenia $v$. manic-depressive insanity (Marneros, 2006) and the unipolar-bipolar dichotomy. Moreover, in agreement with clinical genetic studies (Angst \& Scharfetter, 1990), modern molecular genetic studies demonstrate that there is no clear-cut distinction between schizophrenia and bipolar affective disorder; both clinically and genetically they share many features (Craddock \& Owen, 2005).

Diagnostic concepts of psychiatric syndromes are purely descriptive and cannot 
constitute diseases (Kendell, 1999). The proposed mood spectrum model unifies categorical classification, which is essential, with a dimensional view, which is true to nature; both are needed and both are empirically testable.

\section{REFERENCES}

Akiskal, H. S. (1977) Cyclothymic disorder: validating criteria for inclusion in the bipolar affective group. American Journal of Psychiatry, 134, 1227-1233.

American Psychiatric Association (1994) Diagnostic and Statistical Manual of Mental Disorders (4th edn) (DSM-IV). APA.

Angst, J. (1978) The course of affective disorders. II. Typology of bipolar manic-depressive illness. Archiv für Psychiatrie und Nervenkrankheiten, 226, 65-73.

Angst, J. \& Scharfetter, C. (1990) Schizoaffektive Psychosen - ein nosologisches Ärgernis. In Affektive Psychosen (eds E. Lungershausen, W. P. Kaschka \& R. J. Witkowski), pp. 23-3l. Schattauer.

Angst, J., Adolfsson, R., Benazzi, F., et al (2005) The HCL-32: towards a self-assessment tool for hypomanic symptoms in outpatients. Journal of Affective Disorders, 88, 217-233.

Bleuler, E. (1922) Die Probleme der Schizoidie und der Syntonie. Zeitschrift für die Gesamte Neurologie und Psychiatrie, 78, 373-399.

Craddock, N. \& Owen, M. J. (2005) The beginning of the end for the Kraepelinian dichotomy. British Journal of Psychiatry, 186, 364-366.

JULES ANGST, MD, Zurich University Psychiatric Hospital, Lenggstrasse 31, PO Box 1931, CH-8032 Zürich, Switzerland. Email: jangst@bli.unizh.ch

(First received 8 August 2006, final revision 10 September 2006, accepted II September 2006)

Goldberg, D. (2000) Plato versus Aristotle: categorical and dimensional models for common mental disorders. Comprehensive Psychiatry, 4I, 8-13.

Hirschfeld, R. M. A., Williams, J. B.W., Spitzer, R. L. et al (2000) Development and validation of a screening instrument for bipolar spectrum disorder: the mood disorder questionnaire. American Journal of Psychiatry, I57, $1873-1875$.

Judd, L. L., Akiskal, H. S., Schettler, P. J., et al (2003) A prospective investigation of the natural history of the long-term weekly symptomatic status of bipolar II disorder. Archives of General Psychiatry, 60, 26I-269.

Kendell, R. E. (1999) Much diversity, many categories, no entities (commentary). In Depressive Disorders (eds M. Maj \& N. Sartorius), pp. 52-54.Wiley

Kessler, R. C., Berglund, P., Demler, O., et al (2003) The epidemiology of major depressive disorder. Results from the National Comorbidity Survey Replication (NCS-R). JAMA, 289, 3095-3105.

Kety, S. S., Rosenthal, D., Wender, P. H., et al (1968)

The types and prevalence of mental illness in the biological and adoptive families of adopted schizophrenics. Journal of Psychiatric Research, 6 , S345-S362.

Kleist, K. (1937) Zustandsbilder und Krankheitsarten im Lichte der Gehirnpathologie. Psychiatrischneurologische Wochenschrift, 39, 420-422.
Kleist, K. (1953) Die Gliederung der neuropsychischen Erkrankungen. Monatsschrift für Psychiatrie und Neurologie, 125, 526-554.

Klerman, G. L. (198I) The spectrum of mania Comprehensive Psychiatry, 22, II-20.

Kretschmer, E. (1921) Körperbau und Charakter. Untersuchungen zum konstitutionsproblem und zur Lehre von den Temperamenten (I. Aufl.). Springer.

Lieb, R. (2006) Major depression in adolescents: the role of familial and environmental factors (abstract S7.2) Journal of Affective Disorders, 9I, SI6.

Marneros, A. (2006) Beyond the Kraepelinian dichotomy: acute and transient psychotic disorders and the necessity for clinical differentiation. British journal of Psychiatry, 189, I-2

McCombs, J. S., Ahn, J., Tencer, T., et al (2007) The impact of unrecognized bipolar disorders among patients treated for depression with antidepressants in the fee-for-services California Medicaid (Medi-Cal) program: a 6-year retrospective analysis. Journal of Affective Disorders, in press.

Ösby, U., Brandt, L., Correia, N., et al (200I) Excess mortality in bipolar and unipolar disorder in Sweden. Archives of General Psychiatry, 58, 844-850.

Wing, J. K., Mann, S. A., Leff, J. P., et al (1978) The concept of a 'case' in psychiatric population surveys. Psychological Medicine, 8, 203-217. 